\title{
Multiobjective Process Controllability
}

\section{Analysis*}

\author{
Yi Cao ${ }^{\ddagger \dagger} \quad$ Zhijia Yang ${ }^{\S}$ \\ ${ }^{\ddagger}$ School of Engineering, Cranfield University, UK. \\ $\S$ Department of Energy Engineering, Zhejiang University, China
}

\section{Keywords}

Process Controllability, Multiobjective Optimisation, Linear Matrix Inequality

\begin{abstract}
A new approach for process controllability analysis by using multiobjective optimisation techniques is proposed. Within the approach, a set of performance specifications, such as minimum control error and input effort with closed-loop pole placement are represented as a set of linear matrix inequalities (LMI). The solution to the LMI conditions can be identified as feasible or infeasible. If the solution is feasible there is at least one controller that

*Partially supported by the EPSRC UK grant GR/R57324

${ }^{\dagger}$ To whom correspondence should be addressed. Email:y.cao@cranfield.ac.uk
\end{abstract}


can make the closed-loop system satisfy all performance specifications simultaneously. Therefore, for the process plant, these performance specifications are achievable. Otherwise, they are unachievable. There is a Pareto-optimal set or a trade-off curve in the performance space to separate these two areas. The paper shows that such trade-off curves can be used for process controllability analysis, and therefore, can be applied to control structure selection problems.

\section{Introduction}

The issue of input-output controllability analysis has received increasing attention for a few decades. Input-output controllability is the ability of a plant to achieve acceptable control performance. Various tools and techniques have been developed and are available in the literature to quantify the inherent input-output controllability of a plant (Skogestad and Postlethwaite, 1996). However most of these tools are mainly open-loop controllability indices. Each individual index only addresses one aspect of process controllability, which causes performance limitation, such as input constraints, unstable poles and zeros. It is still an open area to predict performance limitation jointly imposed by these factors. Nonlinear optimisation has been used to predict performance limitation for a plant with input constraints and unstable zeros (Cao et al., 1996). The minimum input usage required to stabilize an unstable plant has been derived by Glover (1986) and recently by Havre and Skogestad (2001). Åström (2000) presented some results for performance limitations in SISO systems. For MIMO systems, the impact of unstable poles and zeros on closed-loop sensitivity and complementary sensitivity functions has been extensively studied by Chen (2000). 
In this paper, performance limitations are presented in trade-off curves, which are used in process controllability analysis. Boyd and Barratt (1991) have revealed that most control system design specifications are affine and convex functions of the controller to be designed. The trade-off curves of these functions have been calculated as Pareto-optimal performance set. As summarised by Scherer et al. (1997), many control performance criteria can be represented as a set of linear matrix inequalities (LMI) ${ }^{1}$ (Boyd et al., 1994). These performance criteria include $H_{\infty}$ and $H_{2}$ norms of certain closed-loop transfer functions and pole placement regions. Using LMI, various, even inconsistent performance requirements can be identified as feasible and infeasible in the performance space. These two areas are separated by the Pareto-optimal performance set. The Pareto-optimal performance set gives a clear picture about what is the achievable performance of a process control system and what kind of performance trade-off is necessary for control design.

The paper is organized as follows. Section 2 introduces the multiobjective control design specifications used in this paper. Two types of Paretooptimal performance curves are designated for multiobjective controllability analysis. These curves are solvable by off-the-shelf software with a small modification. Section 3 provides a complete case study to show the usage of these Pareto diagrams in control structure selection. The paper is concluded in Section 4.

\footnotetext{
${ }^{1}$ A tutorial paper on LMI for chemical process control practitioners is available elsewhere (VanAntwerp and Braatz, 2000).
} 


\section{Multiobjective Process Controllability and LMI}

\subsection{Generalised control configuration}

Control systems can be constructed in different ways, such as: feedback, feedforward, cascade, partial and indirect control. The way a control system constructed is referred to as control configuration. Control configuration has a strong impact on the process controllability. For control structure selection purpose, it is desirable to compare process controllability of different control configurations. Therefore, a generalised control configuration shown in Figure 1 is considered in the paper.

In Figure 1, the block $P$ represents a general plant, whilst block $K$ is a controller. Signals, which link both blocks, are measured output, $y$ and manipulated input, $u$. The signal $w$ represents exogenous inputs, such as disturbances, references, noises and inputs from uncertainties, whilst the exogenous output, $z$, is the control objective. Assume the transfer matrix, $P$ of the general plant is partitioned, according to the inputs and outputs, as follows:

$$
P=\left(\begin{array}{cc}
P_{z w} & P_{z u} \\
P_{y w} & P_{y u}
\end{array}\right)
$$

The state-space form of $P$ is denoted as:

$$
P=\left(\begin{array}{c|cc}
A_{P} & B_{P w} & B_{P u} \\
\hline C_{P z} & D_{P z w} & D_{P z u} \\
C_{P y} & D_{P y w} & D_{P y u}
\end{array}\right)
$$

The control $K$ has the state-space form:

$$
K=\left(\begin{array}{c|c}
A_{K} & B_{K} \\
\hline C_{K} & D_{K}
\end{array}\right)
$$


Then the closed-loop transfer function from the exogenous input, $w$ to the exogenous output, $z$ is:

$$
T_{z w}=P_{z w}+P_{z u} K\left(I-P_{y u} K\right)^{-1} P_{y w}=\left(\begin{array}{c|c}
A & B \\
\hline C & D
\end{array}\right)
$$

where the closed-loop state-space matrices are:

$$
\begin{aligned}
& A=\left(\begin{array}{cc}
A_{P}+B_{P u} R D_{K} C_{P y} & B_{P u} R C_{K} \\
B_{K} C_{P y}+B_{K} D_{P y u} R D_{K} C_{P y} & A_{K}+B_{K} D_{P y u} R C_{K}
\end{array}\right) \\
& B=\left(\begin{array}{c}
B_{P w}+R D_{K} D_{P y w} \\
B_{K} D_{P y w}+B_{K} D_{P y u} R D_{K} D_{P y w}
\end{array}\right) \\
& C=\left(C_{P z}+D_{P z u} R D_{K} C_{P y} \quad D_{P z u} R C_{K}\right) \\
& D=D_{P z w}+D_{P z u} R D_{K} D_{P y w}
\end{aligned}
$$

where $R=\left(I-D_{K} D_{P y u}\right)^{-1}$.

Most control configurations, for example those mentioned above, can uniformly be represented in the generalised configuration. Thus, controllability analysis based on this generalised configuration can directly be applied to control structure selection.

\subsection{Performance Specification}

Many control performance specifications can be expressed as the $H_{\infty}$ norm of certain closed-loop transfer functions. Such functions could be the sensitivity function, $S$, complementary sensitivity function, $T$, the input sensitivity function, $K S$, or more generally, a closed-loop transfer function from $w$ to $z$, i.e. $\left\|T_{z w}\right\|_{\infty}$. Since,

$$
\left\|T_{z w}\right\|_{\infty}=\max _{w} \frac{\|z\|_{2}}{\|w\|_{2}}=\max _{\|w\|_{2}=1}\|z\|_{2}
$$


a performance specification to minimise $\left\|T_{z w}\right\|_{\infty}$ is equivalent to minimise the $L_{2}$-norm of $z$ at the worst case disturbance, $w$. Using the closedloop state-space matrices in equation (4), an $H_{\infty}$ performance condition, $\left\|T_{z w}\right\|_{\infty} \leq \gamma$ (a constant) can be represented in the LMI form as follows:

$$
\left(\begin{array}{ccc}
A^{T} P+P A & P B & C^{T} \\
B^{T} P & -\gamma I & D^{T} \\
C & D & -\gamma I
\end{array}\right)<0, \quad P>0
$$

In $H_{\infty}$ control design, a multiobjective performance specification is usually treated as a mixed sensitivity design problem, such as mixed $S-K S$, or mixed $S-T$ objectives with suitable weighting functions. However, for the controllability analysis purpose, the multiple $H_{\infty}$ norms are better to be considered simultaneously as a multiobjective optimisation problem. The multiobjective $H_{\infty}$ optimisation can be solved by recently developed LMI techniques (Gahinet et al., 1995).

The $H_{\infty}$ norm is a system norm where input and output signals both are in $L_{2}$ space. For control error, this specification is more or less similar to the traditional integral squared error (ISE) performance index. However, to consider the effect of input constraints, it is better to limit the maximal magnitude of the input within its constraints. Therefore, $L_{\infty}$ space is more appropriate than $L_{2}$ in describing the control-input signal, $u$. If disturbance $w \in L_{2}$ and input $u \in L_{\infty}$, then the induced norm from $w$ to $u$ is the generalised- $H_{2}$ norm (Rotea, 1993; Scherer et al., 1997), denoted as $\left\|T_{u w}\right\|_{g}$ :

$$
\left\|T_{u w}\right\|_{g}=\max _{w} \frac{\|u\|_{\infty}}{\|w\|_{2}}=\max _{\|w\|_{2}=1}\|u\|_{\infty}
$$

A performance specification to confine $\left\|T_{u w}\right\|_{g}<\beta$ is equivalent to constrain the magnitude of $u$ less than $\beta$ at the worst case disturbance, $w$. The LMI 
conditions for $\left\|T_{u w}\right\|_{g}<\beta$ (a constant) are given by Scherer et al. (1997) as follows:

$$
\left(\begin{array}{cc}
A^{T} P+P A & P B \\
B^{T} P & -I
\end{array}\right)<0, \quad\left(\begin{array}{cc}
P & C^{T} \\
C & \beta I
\end{array}\right)>0, \quad D=0
$$

Transient response is always involved in performance requirements and can often be achieved by forcing the closed-loop poles into a suitable region. For example, the condition of all closed-loop poles on the left-half plane of $\Re(p) \leq \alpha$ (a negative constant), usually called $\alpha$-stability condition, will force the closed-loop transient response not slower than $e^{\alpha t}$, i.e. the larger the negative value of $\alpha$, the faster the closed-loop transient response. A more general pole region shown in Figure 2 is denoted as $S(\alpha, r, \theta)$ :

$$
S(\alpha, r, \theta)=\{p|\Re(p) \leq \alpha,| p|\leq r, \tan (\theta) \Re(p) \leq-| \Im(p) \mid\}
$$

In this region, $\alpha$ determines the response speed, $\theta$ specifies the minimal damping coefficient of the response and $r$ confines the controller to be well defined (Chilali and Gahinet, 1996). The pole region, $S(\alpha, r, \theta)$ is equivalent to a set of LMI (Chilali and Gahinet, 1996),

$$
S(\alpha, r, \theta)=R(L, M)=\left\{p \mid L+p M+\bar{p} M^{T}<0\right\}
$$

Using matrices, $L=\left\{l_{i j}\right\}$ and $M=\left\{m_{i j}\right\}$, the LMI conditions for the closedloop matrices to satisfy the pole region are (Chilali and Gahinet, 1996):

$$
l_{i j} P+m_{i j} A^{T} P+m_{j i} P A<0, \quad P>0
$$

The multiobjective optimisation problem to be considered in the paper is as follows:

$$
\begin{array}{cc} 
& \gamma=\min _{K}\left\|T_{e w}\right\|_{\infty} \\
\text { subject to: } & \left\|T_{u w}\right\|_{g}<\beta \\
& p \in S(\alpha, r, \theta)
\end{array}
$$


where $e$ is the unit-feedback control error. According to (9) and (11), problem (16) can be explained as that at worst case disturbance with $\|w\|_{2}=1$, a controller $K$ is to be designed to minimise the integral squared control error $\left(\min \|e\|_{2}\right)$ subject to limited input magnitude $\left(\|u\|_{\infty} \leq \beta\right)$ and desired transient response shape $(p \in S(\alpha, r, \theta))$. This problem is ready to be solved by using off-the-shelf software, for example, the MATLAB LMI Control Toolbox (Gahinet et al., 1995). The MATLAB function, hinfmix in the LMI toolbox, originally designed for mixed $H_{2} / H_{\infty}$ problem has been slightly modified for the generalised- $H_{2} / H_{\infty}$ problem in (16). Two multiobjective Pareto diagrams, the minimum $\left\|T_{u w}\right\|_{g}$ against $\alpha$-stability and the minimum $\left\|T_{u w}\right\|_{g}$ against the minimum $\left\|T_{e w}\right\|_{\infty}$ are to be produced by repeatedly calling the modified MATLAB function. The former is to reveal how large control effort is required corresponding to the response speed required whilst the latter gives a global picture what minimal control error is achievable for certain limited input magnitude.

Problem (16) presents a general control design trade-off: minimise control error with minimal control effort. Traditionally, such a trade-off is solved via a combined objective function with properly selected weights, e.g. the Linear Quadratic Gaussian (LQG) problem and mixed sensitivity problem. Separating these two performance requirements into objective function and constraint as shown in (16) is more natural and closer to a practical situation. In addition, the LMI formulations in (10), (12) and (14) are convex. Hence the solution of (16) is global optimal. Thus, index (16) is more suitable than traditional indices such as the LQG for controllability analysis to reveal achievable performance limitation of a plant. 


\section{Case Study}

\subsection{Two-CSTR Process}

The approach for multiobjective controllability analysis is applied to a twoCSTR process. The process is schematically shown in Figure 3. A full description of the system and an eight-state model can be found in (Cao and Biss, 1996). To focus on the control structure selection problem discussed here, constant volume assumption is applied to the process, which leads to a six-state model to be used in the paper. The control problem is to maintain both tank temperatures at desired values in the presence of cooling-water temperature fluctuations within $\pm 10[\mathrm{~K}]$, i.e. $w=\left[T_{c w 1}, T_{c w 2}\right]^{T}$. Three possible control configurations to be considered are:

S1: $u=\left[Q_{I 1}, Q_{I 2}\right]^{T}$, two feed flowrates and $y=\left[T_{o 1}, T_{o 2}\right]^{T}$, two tank outlet temperatures.

S2: $u=\left[Q_{c w 1}, Q_{c w 2}\right]^{T}$, two cooling-water flowrates and $y$ is the same as S1.

S3: $u$ is the same as $\mathrm{S} 2$, but $y$ has two extra secondary measurements, cooling-water outlet temperatures, i.e. $y=\left[T_{o 1}, T_{o 2}, T_{c w o 1}, T_{c w o 2}\right]^{T}$.

The input constraints are

$$
\begin{aligned}
& 0.05 \leq Q_{I 1}+Q_{I 2} \leq 0.8\left[\mathrm{~m}^{3} / \mathrm{s}\right] \\
& 0.05 \leq Q_{c w 1}, Q_{c w 2} \leq 0.8\left[\mathrm{~m}^{3} / \mathrm{s}\right]
\end{aligned}
$$

To cope with the constraints, $Q_{I 1}$ and $Q_{I 2}$ are converted to total flowrate, $Q$ and flowrate ratio, $R$, i.e.

$$
\begin{aligned}
Q & =Q_{I 1}+Q_{I 2} \\
R & =Q_{I 2} / Q
\end{aligned}
$$


The new constraints for $Q$ and $R$ are:

$$
\begin{aligned}
0.05 & \leq Q \leq 0.8\left[\mathrm{~m}^{3} / \mathrm{s}\right] \\
0 \leq R & \leq 1
\end{aligned}
$$

For variable scaling, the manipulated variables are divided by the minimum distance from their steady state value to their boundary. The disturbance variables are divided by $10[\mathrm{~K}]$, whilst the output variables is divided by $1[\mathrm{~K}]$. The final linearised model for configurations S1, S2 and S3 is represented as:

$$
\begin{aligned}
\dot{x} & =A x+B_{i} u+E w \\
y & =C_{i} x
\end{aligned}
$$

where $B_{i}$ and $C_{i}$ are $B$ and $C$ matrices for configurations $\mathrm{S} i$, respectively. The state-space matrices are:

$$
\begin{aligned}
& A=\left(\begin{array}{cccccc}
-17.9751 & -295.8655 & 0 & 0 & 0 & 0 \\
0.0207 & 0.1889 & 0.0704 & 0 & 0 & 0 \\
0 & 0.3879 & -0.8000 & 0 & 0 & 0 \\
0.0977 & 0 & 0 & -18.0088 & -295.8655 & 0 \\
0 & 0.0617 & 0 & 0.0131 & 0.0433 & 0.0589 \\
0 & 0 & 0 & 0 & 0.3787 & -0.6220
\end{array}\right) \\
& \left(B_{1}\left|B_{2}\right| E\right)=\left(\begin{array}{cc|cc|cc}
17.8996 & -13.7811 & 0 & 0 & 0 & 0 \\
-0.0131 & 0.0101 & 0 & 0 & 0 & 0 \\
0 & 0 & -0.0294 & 0 & 0.0137 & 0 \\
17.8636 & 17.8636 & 0 & 0 & 0 & 0 \\
-0.0082 & -0.0082 & 0 & 0 & 0 & 0 \\
0 & 0 & 0 & -0.0235 & 0 & 0.0081
\end{array}\right) \\
& B_{3}=B_{2} \\
& C_{3}=\left(\begin{array}{cccccc}
0 & 362.9950 & 0 & 0 & 0 & 0 \\
0 & 0 & 0 & 0 & 362.9950 & 0 \\
\hline 0 & 0 & 327.5600 & 0 & 0 & 0 \\
0 & 0 & 0 & 0 & 0 & 335.4470
\end{array}\right) \\
& C_{1}=C_{2}=\text { the first two rows of } C_{3}
\end{aligned}
$$




\subsection{Controllability Index Analysis}

The effect of input constraints is normally assessed by the minimum singular value, which are 15.06 for $S_{1}$ and 5.13 for $\mathrm{S} 2$ at steady state, i.e. S1 is better than S2 in terms of input constraints. However, S3 has two secondary measurements, thus this index cannot be directly applied.

On other hand, $\mathrm{S} 1$ is the only configuration, which has two unstable zeros (10.33 and 10.31). Physically, this is because the effect of feed flowrate on tank temperature has two opposite directions - positive via reaction and negative because the feed is colder than the liquid in the tank. Therefore, S2 and S3 are better than S1 in terms of unstable zeros. However, for overall performance, it is difficult to judge which configuration is the best only based on these open-loop controllability indices. Therefore, the multiobjective controllability analysis approach described above is applied to this example.

\subsection{Multiobjective Controllability Analysis}

The multiobjective problem (16) is configured as follows for this particular process: $e$ the control error, i.e. the difference between the two outlet temperatures, $\left[T_{o 1}, T_{o 2}\right]$ and their setpoints; $u$ the manipulated variables, i.e. $\left[Q_{I 1}, Q_{I 2}\right]$ for $\mathrm{S} 1$ and $\left[Q_{c w 1}, Q_{c w 2}\right]$ for $\mathrm{S} 2$ and $\mathrm{S} 3$ respectively; $w$ the disturbances, i.e. the cooling water inlet temperatures, $\left[T_{c w 1}, T_{c w 2}\right]$. The closedloop poles region is defined as $r=20$ and $\theta=77.6^{\circ}$ (minimum closed-loop damping coefficient about 0.215 ) with $\alpha$ fixed to -0.5 (maximum closed-loop time constant about $2[\mathrm{~s}]$ ) or varying. To force zero error at steady-state, an integrator is inserted into each error channel and will be merged into the controller designed. Based on these conditions, the multiobjective Pareto diagrams are produced and shown in Figure 4. 
In order to explain the controllability behaviour presented in Figure 4, open-loop poles and zeros of all configurations are listed as follows:

$$
\begin{array}{rllll}
\text { poles: } & -17.8, \quad-17.6, \quad-0.84,-0.67,-0.13-0.11 \\
\text { zeros of } \mathrm{S} 1: & 10.33, \quad 10.31, \quad-0.8,-0.62 \\
\text { zeros of } \mathrm{S} 2: & -18.01, \quad-17.98 \\
\text { zeros of } \mathrm{S} 3: & \text { none }
\end{array}
$$

Comparing open-loop poles with Figure 4 (a) shows that input magnitude required for $\alpha$-stability is depend on the location of the open-loop poles, which are on the right of the $\alpha$-line. The longer the distance of these poles to the $\alpha$-line, the larger the input magnitude required. However, the input magnitude required is also dependent on the controllability of these poles. To move a less controllable pole requires larger control effort. For S1, the open-loop zeros of -0.8 and -0.62 is very close to open-loop poles, -0.84 and -0.67 . Therefore, these two poles have less controllability in S1 than the same poles in other configurations. This causes the sharp increase of input magnitude required $\left(\left\|T_{u w}\right\|_{g}\right)$ of $\mathrm{S} 1$ when $-\alpha>0.6$ (see Figure 4 (a) from $\alpha=-0.6$ towards left).

The results also show that the achievable performance of S1 in terms of $\left\|T_{e w}\right\| \infty$ has a low bound about 0.003 (vertical part of the solid-line in Figure $4(\mathrm{~b}))$. The lower bound is due to its unstable zeros, thus is independent of input constraints.

However, S1 is still the best configuration when $-\alpha<0.6$ and $\|u\|_{\infty}<1$ (i.e. $\left\|T_{u w}\right\|_{g}<1$ in the figures). It is also shown that the nonsquare configuration, S3 does improve the controllability by introducing extra measurements into configuration S2. It can achieve almost the same performance as S1 within the input constraints (Figure 4 (b) at $\left\|T_{u w}\right\|_{g}=1$ ). If the input 
constraints were permitted to increase slightly, S3 would even be better than S1. This observation is verified by the simulation results (see Figures 5).

\subsection{Nonlinear Simulation}

To verify the results obtained in multiobjective controllability analysis, a simulation has been carried out on the original nonlinear model (Cao and Biss, 1996). The controllers $K_{1}, K_{2}$ and $K_{3}$ given in equations (29), (30) and (31) are designed for control configurations S1, S2 and S3 respectively. These controllers are calculated to achieve performance specification of $\left\|T_{e w}\right\|_{\infty}$ to the level corresponding to $\left\|T_{u w}\right\|_{g}=1$ in Figure 4(b). The actual maximal input magnitudes observed in the simulation match the $\left\|T_{u w}\right\|_{g}$ values predicted in Figure 4(b) (see Figure 6).

$$
\begin{aligned}
K_{1}= & \left(\begin{array}{c|cccccc|cc}
A_{K 1} & B_{K 1} \\
\hline C_{K 1} & D_{K 1}
\end{array}\right) \\
& =\left(\begin{array}{cccccccccc}
-3.87 & 32.82 & -7.04 & 1.26 & 0.88 & -0.45 & -32.88 & 2.62 & 0 & 0 \\
-33.08 & -11.05 & 0.31 & 5.43 & -1.77 & 0.24 & -45.92 & -4.12 & 0 & 0 \\
6.33 & 1.77 & -7.12 & 26.06 & -0.28 & -1.71 & 7.23 & -33.08 & 0 & 0 \\
-0.68 & -5.57 & -25.98 & -14.60 & 1.14 & 2.54 & -4.06 & -33.47 & 0 & 0 \\
-0.94 & -1.48 & 1.13 & 0.97 & -0.90 & -0.02 & -3.62 & 1.88 & 0 & 0 \\
0.34 & 0.91 & 1.41 & 2.59 & -0.04 & -0.94 & 1.64 & 3.39 & 0 & 0 \\
0 & 0 & 0 & 0 & 0 & 0 & 0 & 0 & 1 & 0 \\
0 & 0 & 0 & 0 & 0 & 0 & 0 & 0 & 0 & 1 \\
\hline-15.81 & 15.41 & -18.02 & 14.26 & 0.93 & -2.07 & -46.17 & -35.40 & 0 & 0 \\
8.63 & -16.65 & -6.80 & 10.41 & -1.64 & -0.67 & 27.67 & -31.41 & 0 & 0
\end{array}\right)
\end{aligned}
$$




$$
\begin{aligned}
& K_{2}=\left(\begin{array}{c|c}
A_{K 2} & B_{K 2} \\
\hline C_{K 2} & D_{K 2}
\end{array}\right) \\
& =\left(\begin{array}{cccccccc|cc}
-0.32 & -19.71 & 0.05 & 0.02 & 1.12 & -0.01 & -19.43 & 0.17 & 0 & 0 \\
19.70 & -10.39 & 0.19 & 0.05 & 5.75 & -0.08 & 100.71 & 3.60 & 0 & 0 \\
-0.05 & -0.00 & -0.52 & 11.78 & -0.21 & -1.59 & -0.84 & 13.83 & 0 & 0 \\
-0.09 & 0.05 & -11.79 & -10.06 & 0.18 & 6.40 & -2.12 & 50.06 & 0 & 0 \\
-1.12 & 5.75 & 0.03 & -0.02 & -8.98 & 0.04 & -30.90 & -1.44 & 0 & 0 \\
0.03 & -0.01 & 1.60 & 6.40 & -0.09 & -8.11 & 0.76 & -18.69 & 0 & 0 \\
0 & 0 & 0 & 0 & 0 & 0 & 0 & 0 & 1 & 0 \\
0 & 0 & 0 & 0 & 0 & 0 & 0 & 0 & 0 & 1 \\
\hline 6.79 & 35.24 & -0.11 & -0.87 & -10.81 & 0.42 & 34.60 & -0.22 & 0 & 0 \\
0.21 & 0.96 & -3.08 & 11.11 & -0.44 & -4.14 & 0.37 & 17.86 & 0 & 0
\end{array}\right) \\
& K_{3}=\left(\begin{array}{l|l}
A_{K 3} & B_{K 3} \\
\hline C_{K 3} & D_{K 3}
\end{array}\right) \\
& =\left(\begin{array}{ccccc|cccc}
-5.15 & -0.56 & -3.42 & -0.33 & -7.19 & 0 & 0 & 0.11 & -0.65 \\
-0.64 & -0.61 & -0.46 & 1.88 & -0.89 & 0 & 0 & -1.04 & -0.13 \\
-2.84 & -0.43 & -2.76 & 0.14 & -2.91 & 0 & 0 & 0.03 & 0.98 \\
0 & 0 & 0 & 0 & 0 & 1 & 0 & 0 & 0 \\
0 & 0 & 0 & 0 & 0 & 0 & 1 & 0 & 0 \\
\hline 0.13 & -0.75 & -0.00 & 20.89 & 0.24 & 0 & 0 & 0 & 0 \\
-1.60 & -0.20 & -0.68 & 0.01 & 13.17 & 0 & 0 & 0 & 0
\end{array}\right)
\end{aligned}
$$

The simulation results are shown in Figures 5 and 6 . The figures clearly indicate that the predictions made from multiobjective controllability analysis are correct: $\mathrm{S} 1$ is the best configuration in terms of control accuracy and response speed. By introducing secondary measurements, S3 can achieve almost the same performance as S1. Due to the nonlinearity of the process, in the case of negative disturbance, S3 is even better than S1. Comparing input response of $\mathrm{S} 2$ and $\mathrm{S} 3$ in Figure 6, it is shown that the control effort required for disturbance rejection is slightly reduced by using secondary measurements (Figure 6). 


\section{Conclusions}

The proposed approach for multiobjective controllability analysis is able to identify performance limitation imposed by multi-factors, such as unstable zeros and input constraints. It is also suitable for more sophisticated configurations, such as nonsquare, cascade and two degrees-of-freedom control. The produced Pareto diagrams can be directly used for control design trade-off. The generalised- $H_{2}$ norm is better than $H_{\infty}$ norm to describe input with input constraints. The enforced closed-loop pole region makes the closed-loop time response more predictable.

\section{References}

Åström, K. J. (2000). Limitations on control system performance. European Journal on Control 6(1), 2-10.

Boyd, S., L. E. Ghaoui, E. Feron and V. Balakrishnan (1994). Linear Matrix Inequalities in System and Control Theory. SIAM. Philadelphia.

Boyd, S. P. and C. H. Barratt (1991). Linear Controller Design, Limits of Performance. Prentice Hall Information and System Sciences Series. Prentice Hall. Englewood Cliffs, NJ.

Cao, Y. and D. Biss (1996). An extension of singular value analysis for assessing manipulated variable constraints. Journal of Process Control 6(1), 37-48.

Cao, Y., D. Biss and J. D. Perkins (1996). Comparison of two techniques for assessing input-output controllability in the presence of manipulated variable constraints. Computers Chem. Engng. 20(4), 337-346. 
Chen, J. (2000). Logarithmic integrals, interpolation bounds, and performance limitations in MIMO feedback systems. IEEE Trans. Autom. Control 45(6), 1098-1115.

Chilali, M. and P Gahinet (1996). $H_{\infty}$ design with pole placement constraints: an LMI approach. IEEE Trans. Autom. Control 41, 358-367.

Gahinet, P., A. Nemirovski, A. Laub and M Chilali (1995). The LMI Control Toolbox. The MathWorks, Inc.

Glover, K. (1986). Robust stabilization of linear-multivariable systems - relations to approximation. International Journal of Control 43, 741-766.

Havre, K. and S. Skogestad (2001). Achievable performance of multivariable systems with unstable zeros and poles. International Journal of Control 74, 1131-1139.

Rotea, M.A. (1993). The generalized $H_{2}$ control problem. Automatica 29, 373-385.

Scherer, C., P. Gahinet and M. Chilali (1997). Multiobjective outputfeedback control via LMI optimization. IEEE Trans. Autom. Control 42, 896-911.

Skogestad, S. and I. Postlethwaite (1996). Multivariable Feedback Control. Analysis and Design. John Wiley \& Sons. Chichester.

VanAntwerp, J.G. and R.D. Braatz (2000). A tutorial on linear and bilinear matrix inequalities. J. Process Control 10(4), 363-385. 


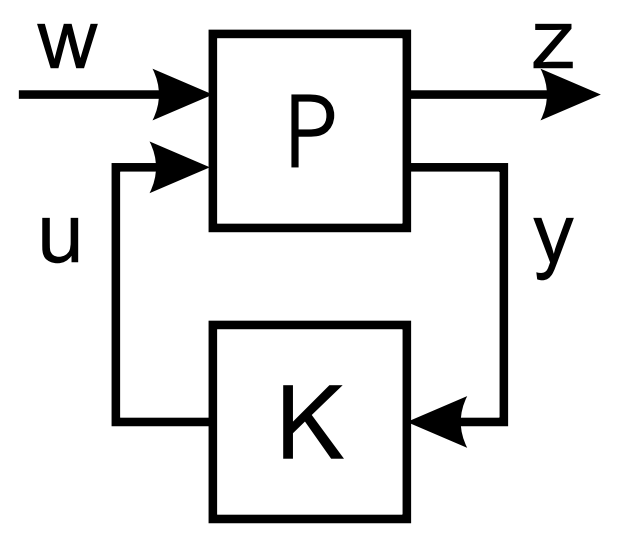

Figure 1: Generalised Control System

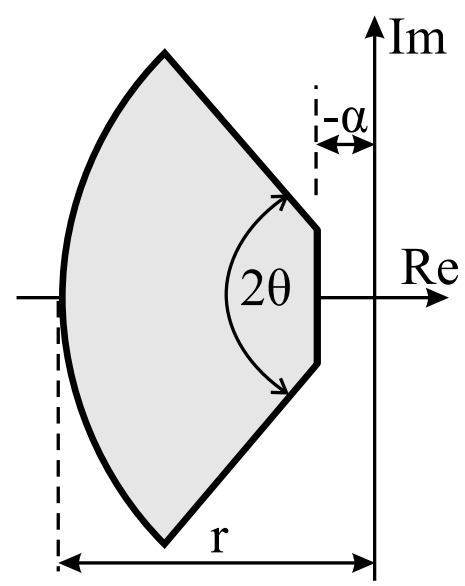

Figure 2: Pole Region 


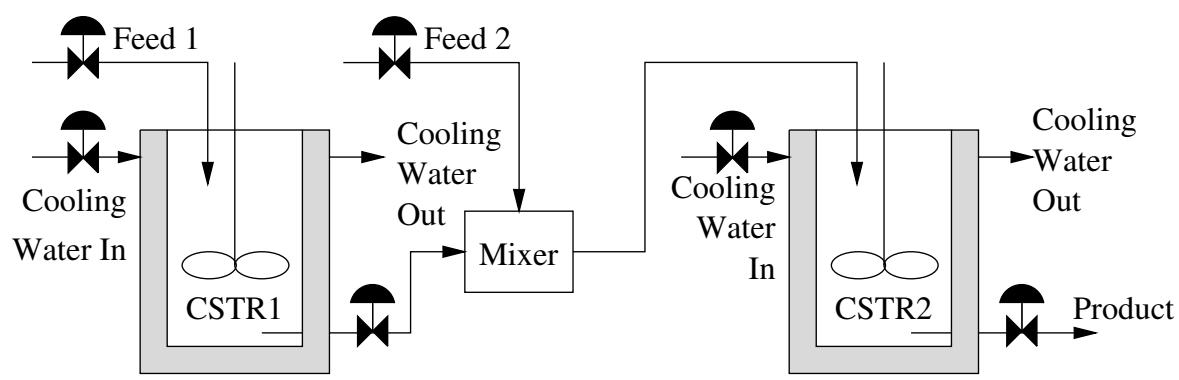

Figure 3: Two-CSTR Plant

(a) Pareto Set: $\mathrm{H}_{2} \vee \alpha$-stability

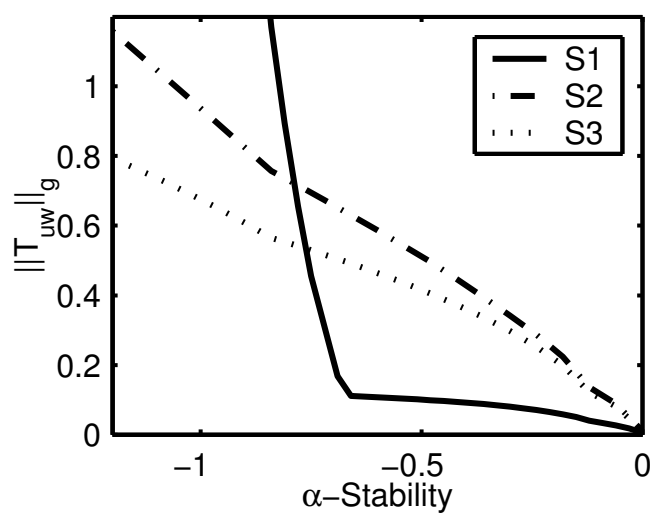

(b) Pareto Set: $\mathrm{H}_{2} \vee \mathrm{H}_{\infty}$

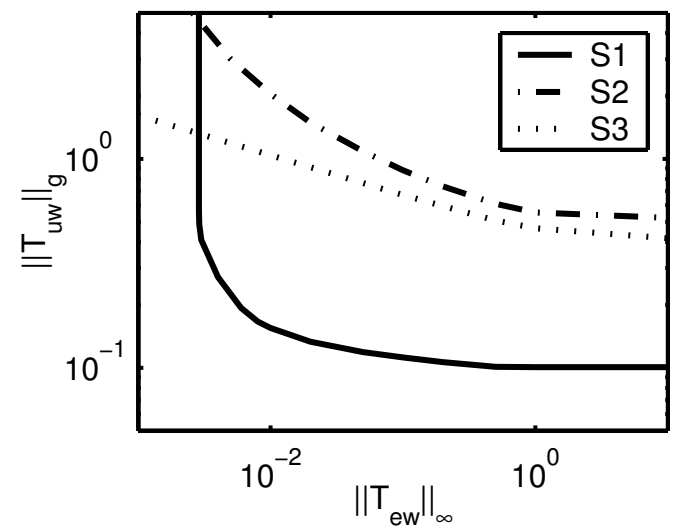

Figure 4: Pareto Diagrams with Pole Region $r=20, \theta=77.6^{\circ}$ and $\alpha=-0.5$ in (b) 
(a) $T_{01}$ Response to $T_{c w}+10[K]$

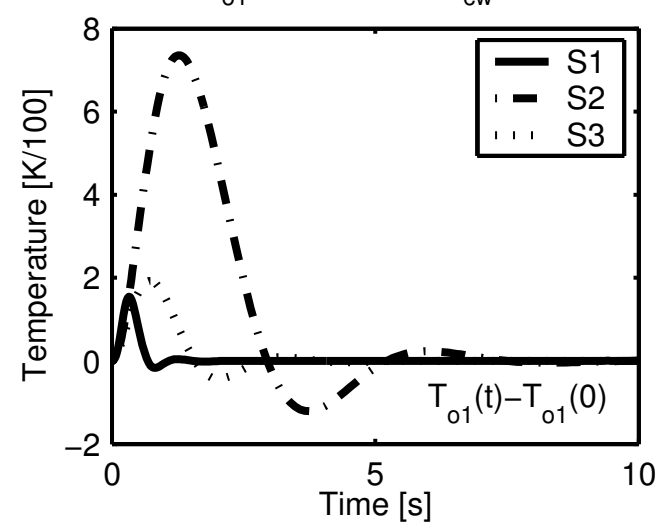

(c) $T_{01}$ Response to $T_{c w}-10[K]$

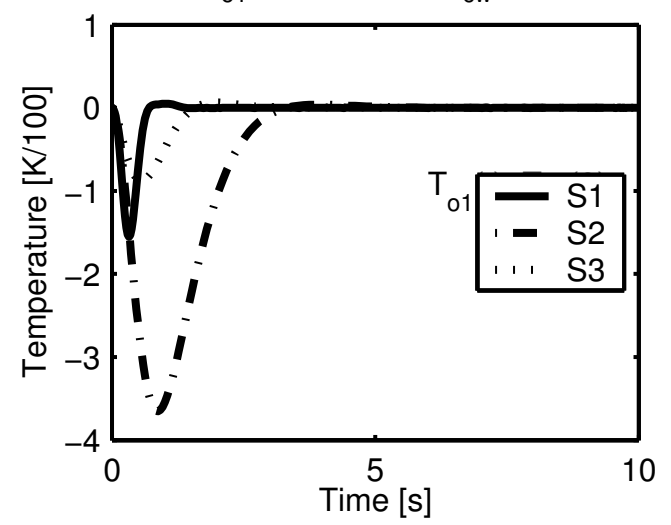

(b) $\mathrm{T}_{02}$ Response to $\mathrm{T}_{\mathrm{cw}}+10[\mathrm{~K}]$

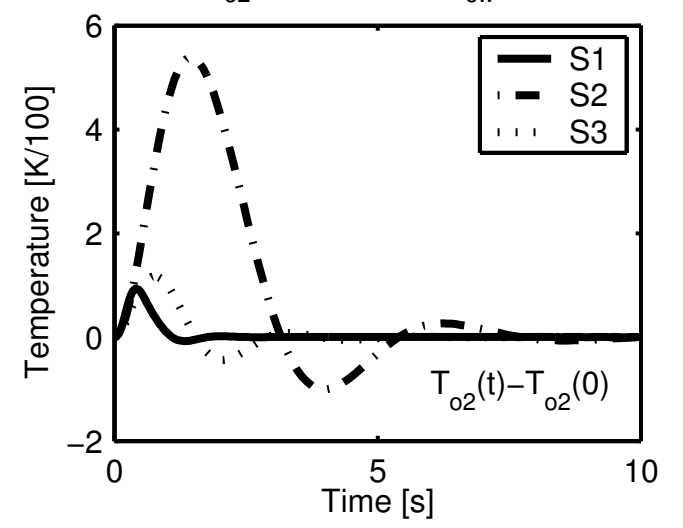

(d) $\mathrm{T}_{02}$ Response to $\mathrm{T}_{\mathrm{cw}}-10[\mathrm{~K}]$

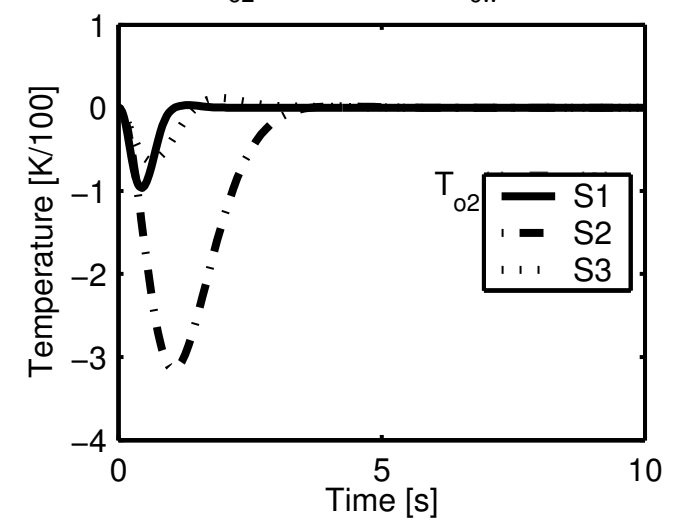

Figure 5: Output Response 
(a) S1: Q Response to $T_{c w}$

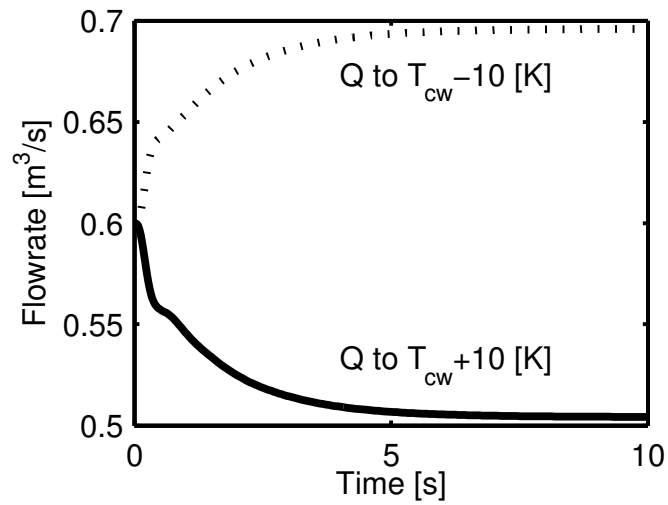

(c) $Q_{\mathrm{cw}}$ Response to $\mathrm{T}_{\mathrm{cw}}+10[\mathrm{k}]$

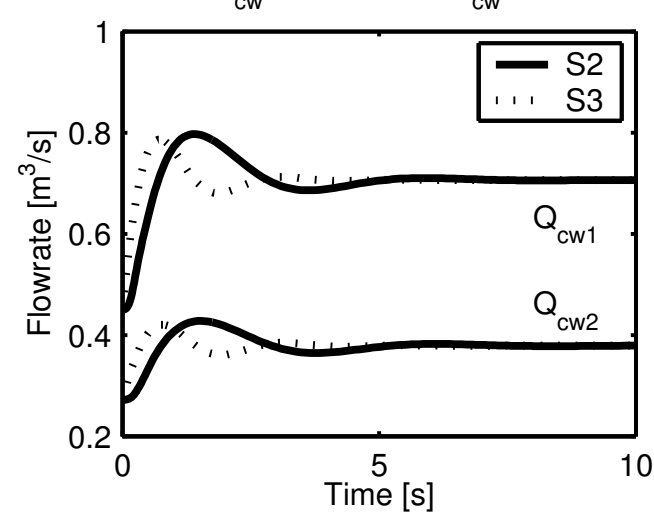

(b) S1: R Response to $T_{c w}$

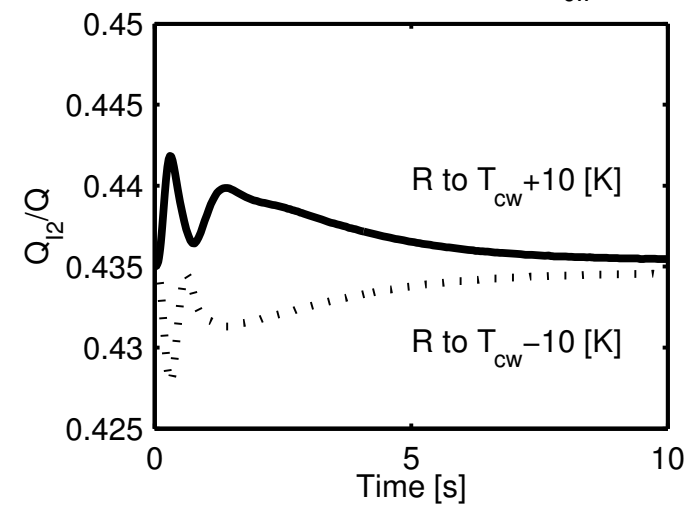

(d) $Q_{c w}$ Response to $T_{c w}-10[k]$

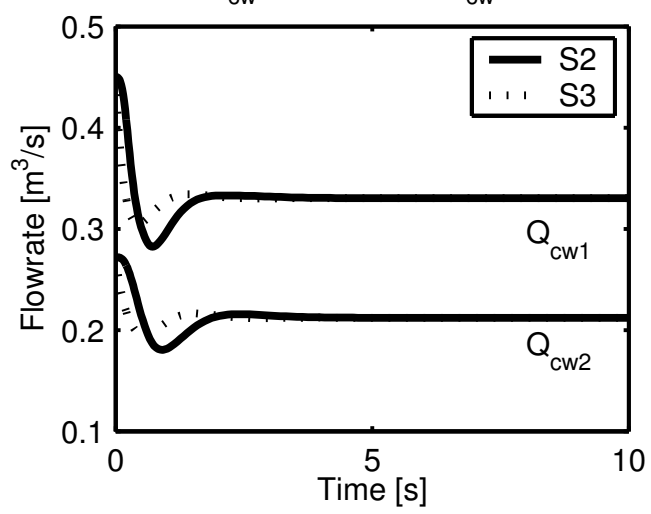

Figure 6: Input Response 